\title{
Use of motivational and educational techniques in behavioral health patients to impact self-care and emergency department visit rates
}

\author{
Saadia A. Basit, Marshall J. Getz, Heather Chung * \\ Houston Methodist Hospital, Houston, United States
}

Received: July 14, 2015

DOI: $10.5430 /$ jnep.v6n6p48
Accepted: November 30, $2015 \quad$ Online Published: February 16, 2016

URL: http://dx.doi.org/10.5430/jnep.v6n6p48

\begin{abstract}
Background and objective: People with mental health and substance use disorders present with multiple medical comorbidities, social and legal issues. Due to these care complexities, this patient population has high rates of hospital readmissions and emergency department (ED) visits. Patients with mental health disorders require integrated care, which is the coordination of physical and behavioral health care. These patients may benefit from various educational techniques and counseling including the Teach Back Method (TBM) and motivational interviewing (MI). Houston Methodist Hospital (HMH) Behavioral Health Transition of Care Program, a quality improvement program, utilizes educational tools and counseling techniques during inpatient and post-discharge phases to improve care coordination in patients with behavioral health conditions. One of the goals of the program is to contribute to a reduction in the behavioral health/substance abuse diagnosis ED visit rate at the participating HMH system hospitals. For the first year (April 2014 through March 2015), the goal was to reduce the behavioral health ED visit rate by 5\% from baseline (October 2013 through April 2014). This paper aims to 1) provide evidence to enhance motivation and establish partnership with patients, 2) report on the Behavioral Health Transition of Care Program's use of motivational and educational techniques, 3) describe the program's patient demographics from June 2014 through March 2015, 4) report program performance data, and 5) report ED visit data of patients with a primary or secondary behavioral health or substance abuse diagnosis.

Methods: Data for two of three HMH hospitals participating in the Behavioral Health Transition of Care Program are reported. Staff members carrying out interventions include social workers, educators, nurse practitioners, and a clinical pharmacist. Patients are eligible for inclusion in program interventions if they have a current or previous mental health or substance abuse disorder and are at high risk for readmission (determined by the Discharge Decision Support System [D2S2] conducted by the floor nurse). Social workers are consulted on high risk patients to conduct a Personal Health Record (PHR) and Morisky Medication Adherence Scale (MMAS-8) and enroll patients in the post-discharge interventions (telephone calls, home visits, or both). The clinical pharmacist is consulted on inpatients with a low MMAS- 8 for coaching and medication education. After discharge, enrolled patients receive follow-up automated telephone calls. Educators call patients with post-discharge issues reported via these calls. Nurse practitioners conduct one to two home visits over the 30-day post-discharge period. Aggregate data was obtained using reports obtained for quality-improvement purposes. Descriptive statistics are reported.

Results: Of the 2,330 high risk encounters at HMH and San Jacinto Methodist (SJ) over June 2014 through March 2015, the average age was 55.8 years old, $4.68 \%$ encounters were insured by Medicaid, and the average D2S2 score (range: 0-11) was 4.4. Social workers completed PHR on $73.61 \%$ of the encounters and $13.48 \%$ of the discharged encounters had home visits within 30 days after discharge. There was a $4.6 \%$ reduction in the behavioral health ED visit rate from baseline to first year.

Conclusions: HMH implemented a Behavioral Health Transition of Care Program that uses MI and the TBM to facilitate in reduction of ED utilization by behavioral health patients. Although the goal of 5\% ED visit rate reduction was not achieved, various contributing factors such as a high demand but limited supply of primary care providers may impact the rate.
\end{abstract}

Key Words: Emergency department visits, Motivational interviewing, Patient education, Teach-Back Method, Transitional care

*Correspondence: Heather Chung; Email: HChung@ @oustonmethodist.org; Address: Houston Methodist Hospital, Houston, United States. 


\section{INTRODUCTION}

\subsection{Readmissions}

The revolving door of chronically ill patients utilizing emergency departments (EDs) for non-emergent health conditions is an ongoing problematic expense for the United States. The federal government estimates that approximately 2 million readmissions occur each year at a ratio of 1:5 Medicare patients returning to the hospital within 30 days of discharge. ${ }^{[1]}$ Since 2012, hospitals with readmission rates exceeding the national average for certain conditions suffer a reduction in their Medicare reimbursements. The cut in funding includes patients who are readmitted and for all of the hospital's Medicare patients who receive care. Initially, the penalty was up to $1 \%$ of reimbursements; however, in 2015 it rose to $3 \%$. Currently, $78 \%$ of the nation's hospitals are being fined for high readmissions each month. That is up from $64 \%$ in 2013 and $66 \%$ in 2014, according to research by the Henry J. Kaiser Family Foundation (2015) using data from the Centers for Medicare and Medicaid Services (CMS). Texas has the second highest number of hospitals penalized with 213 , or 56 percent of its total, the analysis found. The Medicare cost associated with these staggering readmission rates is estimated at \$26 billion annually. CMS estimates that as much as $\$ 17$ billion of the cost for readmission are from potentially avoidable readmissions. ${ }^{[1]}$ These penalties to hospitals created financial incentives to fix the problem. The Houston Methodist Hospital (HMH) has two Delivery System Reform Incentive Payment (DSRIP) 1,115 Waiver projects aimed at reducing readmissions for patients who have behavioral health conditions. The Behavioral Health Transition of Care Program, one of the DSRIP 1,115 Waiver projects at $\mathrm{HMH}$, is designed with a set of actions to ensure the coordination and continuity of health care as patients return to their community. The program utilizes an individualized yet comprehensive approach to care management. The interdisciplinary team that specializes in chronic disease management supports patients in symptom management, medication adherence, and post discharge follow-up appointments.

\subsection{Transition of care}

When a patient discharges from one care setting to another, there is an increased risk of adverse events and hospitable readmissions and ED visits. With hospitalization, patients and caregivers are faced with a plethora of new information including medications, diagnoses, and self-care instructions. Often times, complex health information is provided to the patient on the day of hospital discharge. Uncoordinated care yields poor patient-centered outcomes and economic burden. In efforts to smooth transitions of care, health care systems are implementing continuity of care interventions which serve to empower patients and their caregivers. ${ }^{[2]}$

Published by Sciedu Press

\subsection{Complexities of patients with co-occurring behav-} ioral and physical comorbidities

In general, complexity of care increases in patients with behavioral and medical co-morbidities. Research has shown that people with serious mental illness have high rates of co-occurring medical conditions and have a reduced life expectancy compared to the general population. ${ }^{[3]}$ The higher mortality rate in this population is related to physical conditions, especially cardiovascular disease, diabetes and other treatable medical conditions. The mentally ill are more likely than others to not adhere to their medications, be homeless, socially isolated, and negligent about self-care. ${ }^{[4,5]}$ A 2011 report by the Texas Health and Human Services Commission (HHSC) found that patients with serious mental illnesses were among the top five severity-adjusted diagnosis-related groups by volume for readmission and that patients with mental health disorders admitted with medical-surgical conditions were $83 \%$ more likely to be readmitted. ${ }^{[6]}$ Healthrelated information is currently not provided in a patientcentered way and rarely provides ways to address symptoms, manage medications, and how to answer ongoing health concerns. ${ }^{[7]}$ Patients with mental health disorders may benefit from additional coaching and counseling on medications and diagnoses during and after hospitalization.

\subsection{The challenge}

In 2011, an estimated $80 \%$ of adults $18-64$ years old visited the ED due to lack of a provider. ${ }^{[8]}$ Furthermore, studies have shown that $30 \%$ of all ED visits in the United States are non-urgent. ${ }^{[9]}$ The social realities of many contemporary American communities frequently complicate hospital functioning, prompting John O'Shea of The Heritage Foundation to suggest that poverty and other social stressors of these patients may compromise their ability to address emergencies. ${ }^{[10]}$ The remaining force is the financial operation of the hospital and pressures to have positive profit margin. EDs frequently provide care to patients in need of a medication refill or minor medical problem, rather than a true emergency. Use of the pejorative phrase "frequent flyers" is an unfair label as patients with lingering symptoms may feel the need for a longer length of stay and those with complex medication regimens may not be able to adhere to their medications.

\subsection{Coleman model of transition of care}

Given the difficulties hospitals face in serving their communities and maintaining their tight financial margins, a model designed to reduce ED visits could prove valuable. If a clinical team could identify contributing factors to superutilization, a strategy could be initiated to address those specific patient risk factors. Research suggests a variety of approaches to address patients at high risk for readmission 
including setting goals informed by the self-determination theory and the power of collaboration between the patient and health care team. ${ }^{[11,12]}$ Simoneau and Bergeron studied substance abusers in treatment, and found that their "initial motivation" accounted for less than one-third of their progress 45 days later. ${ }^{[11]}$ This statistic means that their patients' sense of personal agency was not a weighty factor in terms of other components of the treatment process.
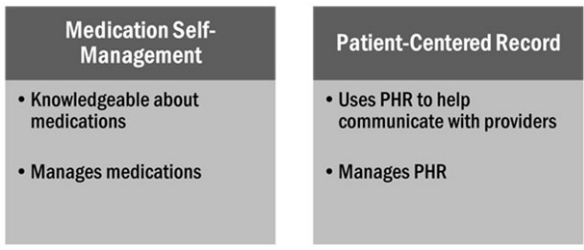

Figure 1. Coleman Four Pillars

1.6 HMH Behavioral Health Transition of Care Program

HMH Behavioral Health Transition of Care Program, a quality improvement initiative, utilizes the Coleman Model in the inpatient and post-discharge phases to improve care coordination and tailor interventions for patients with a primary or secondary mental health/substance use diagnosis at high risk for readmission or ED visit. The program's primary aims are to design, implement, and evaluate interventions to improve care transitions for individuals with a primary or secondary mental health/substance abuse diagnosis. This milestonebased program is established in three of the seven $\mathrm{HMH}$ hospitals: one hospital in the Texas Medical Center (HMH Main, 824 beds) and two community hospitals serving areas surrounding Houston (Willowbrook, 241 beds and San Jacinto, 375 beds). This paper will report data on the hospitals with favorable outcomes: HMH Main and SJ. The program was started at HMH Main and Willowbrook in June 2014 and in San Jacinto in September 2014. A complete description of the program is included in a previously published article. ${ }^{[15]}$ This program aims to reduce the target population's ED visit rate by $5 \%$ over a 1 -year period and by $10 \%$ over a 2 -year period. The baseline ED visit rate of the target population, $31.5 \%$, for HMH and San Jacinto was established October 1, 2013-March 31, 2014. Interventions including the Coleman Model, teach back methods (TBM) and motivational interviewing (MI) are utilized in this program to empower patients to successfully manage their medical and psychiatric conditions after hospital discharge.

\subsection{Scenario: Behavioral health transition of care pro- gram patient}

At the end of the shift report, Nurse Jane reports that Mrs. Smith, a 65-year-old new admission for chest pain, has a
The Coleman Model of Transition of Care is based on the idea of empowering the patient to self-care. ${ }^{[13]}$ Wasson and Coleman found that $75 \%-89 \%$ of self-rated highly confident people versus $40 \%$ of patients with lower confidence adhered to an exercise regimen. ${ }^{[13]}$ The Coleman Model is based on four pillars: medication self-management, patient-centered record, follow-up, and red flags (see Figure 1). ${ }^{[14]}$
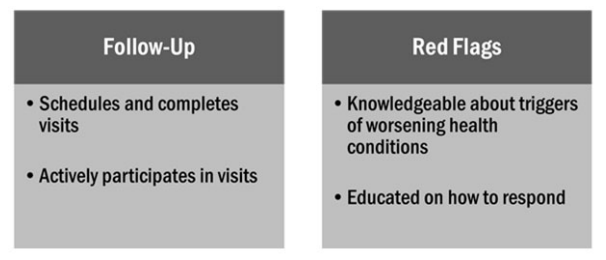

hemoglobin A1c of $12 \%$. The patient is not willing to stop drinking 6 to 10 sodas daily. Nurse Betty responded, "These noncompliant diabetic patients always overeat and are frequently readmitted to the hospital since they don't care about their health." The nurses overlooked the documentation in the history and physical that the patient was depressed and grieving the recent loss of her son. Mrs. Smith's daughter shared that her mother was admitted for the same symptoms 5 times between January and March. They have no complaints regarding the medical care received at the hospital, however the sheer number of readmissions is taking a mental and physical toll on this elderly patient. Patients with multiple psychiatric and medical comorbidities are often quickly labeled as "noncompliant". The ubiquitous labeling of patients as "noncompliant" highlights the ignorance of the health care team to the struggles that patients go through and allows judgment to be placed when patients are not achieving a health-related goal decided by the health care team. Rather than labeling patients as "noncompliant", health professionals need to care for patients holistically, keeping in mind the psychosocial factors impacting self-care. The health care team must consider that there is a scarcity of knowledge on disease and medication self-management as well as limited motivation among these "noncompliant" patients. A paradigm shift in addressing non-adherent patients is in order. Exploring patient beliefs and attitudes about their health and medications may reveal resolvable barriers. Patients cannot be adherent to medical instructions and medications unless they are actively engaged in their care. A caregiver should be present for those patients that may not be able to engage in counseling or coaching due to cognitive deficits possibly stemming from neurological or psychiatric disorders.

ISSN 1925-4040 E-ISSN 1925-4059 


\subsection{Reasons for change}

Despite the striking amount of evidence supporting the efficacy of motivation-focused interventions, their application in the United States has primarily taken place in research settings. A contributing factor is the notion that low motivation, denial and opposition are often viewed as inherent characteristics of patients with mental health or substance abuse disorders. Patients' lack of motivation to change has often been the focus of interest and frustration for clinicians. The cognitive-behavioral burden of the motivational approach requires a paradigm shift in addressing these "noncompliant" patients. This treatment style places greater responsibility on the health care team, whose job is now expanded to include engendering motivation. ${ }^{[16]}$ According to the Diagnostic and Statistical Manual of Mental Disorders, diagnostic criteria for psychiatric disorders such as schizophrenia include negative symptoms such as apathy, inability to enjoy pleasure, and/or amotivation. ${ }^{[17]}$ The challenges for the health care team involve screening and identifying patients' underlying abilities and tailoring treatment interventions according to clients' stage of readiness for change. Patient-centeredness requires profound change in health care design as well as a shift in control from providers to patients. ${ }^{[18]}$ Traditional information sharing consists of standard medical instructions provided to patients and caregivers. Recent evidence reveals the need for health care providers to create a paradigm shift to empower patients in disease self-management. By utilizing an individualized patient-centered, stage-based approach, clinicians may be more effective in behavior modification, even in brief hospital or clinic visits. ${ }^{[19]}$ This paper aims to 1) provide evidence to enhance motivation and establish partnership with patients, 2) report on the Behavioral Health Transition of Care Program's use of behavioral change and educational techniques, 3) describe HMH and SJ patient demographics from June 2014 to March 2015, 4) report HMH and SJ program performance data, and 5) report HMH and SJ ED visit data of patients with a primary or secondary behavioral health or substance abuse diagnosis.

\section{Literature REVIEW}

\subsection{Teach Back Methods}

A majority of this paper describes methods to elicit change in patients, however in health care, sharing knowledge of new diagnoses and medications is often done to facilitate the process in which patients provide self-care. A fundamental tenet of education requires some form of testing, or eliciting a response from the student to show that the student has understood the material just taught. ${ }^{[20]}$ At that point, the student owns the content, and hopefully, can make full use of it. Testing in the education sphere becomes the basis for grades, promotion and academic standing. In the health care world, the stakes may be higher. A patient's understanding of his or her illness may well make the difference between symptoms and relief, maintenance and relapse, and life and death. Many illnesses require lifestyle changes that patients, particularly those in the precontemplative stage of change, do not want to address. ${ }^{[21]}$ Medications may cause financial concerns or side-effects. For both families and patients, being able to discuss and receive answers in appropriate ways can make the hospitalization and post-discharge periods better experiences. ${ }^{[22]}$

Health care staff face a variety of challenges in educating patients and families sufficiently enough to ensure that they will be able to provide at least an adequate level of postdischarge care. Professionals use the term "health literacy" to describe a lay person's comprehension of diagnosis, treatment, and outcome. Patients and their families may lack the education levels, awareness or even the intellectual capacity to understand an illness. External social factors, ranging from poverty and homelessness to legal issues, may easily impede self-care. ${ }^{[23,24]}$ Patients may still be ill, anxious, and distracted when they leave the hospital and unable to grasp their post-discharge instructions.

The Teach Back Method (TBM) has empirical support for guiding patients or caregivers in treatment and post-discharge care. ${ }^{[25-27]}$ TBM provides a tool for educating patients in a simple yet highly effective manner. ${ }^{[28]}$ The United States government recognizes TBM as a safety measure, in which knowledge and understanding become influential factors in patient care. ${ }^{[29]}$ Like any teacher, the health care staff expect to be able to determine if the patient has mastered the skill or material. Rather than the nurse or pharmacist asking patients "did you understand?" and taking them at their word, patients must recall what they have learned. White et al. reported that TBM when used in cardiac patients decreases the likelihood of cardiac-related hospital readmission. ${ }^{[27]}$ In contrast, Tamariz et al. described one study's success using an intensive conversation with patients, without specifying employment of TBM. ${ }^{[30]}$ In that particular study, patients receiving extended teaching time showed an $8 \%$ lead over patients who were not as well-instructed. White's team also found value in a longer training time as well. ${ }^{[27]}$ According to $\mathrm{Xu}$, a patient or family member may be able to understand and follow a complicated medication regimen after being taught with the TBM. ${ }^{[31]}$ Reports show that the TBM leads to greater patient adherence when paired with simple written or audiovisual materials. ${ }^{[29]}$ Black et al. found that patients who received the TBM along with discharge instructions on electronic heart-monitoring equipment indicated better understanding of both that equipment and their post-discharge 
care better than other patients. ${ }^{[32]}$ While there is a wealth of evidence on the TBM in the medical population, evidence on the TBM in the psychiatric population remains scant.

\subsection{Self-care}

Dorothea Orem's Self-care Deficit Nursing Theory indicates that self-care, or the practice of activities that adults initiate to maintain health, life and well-being, is usually initiated voluntarily. ${ }^{[33]}$ Ryan et al. affirmed that in order for healthrelated changes to be successfully initiated and sustained, patients need to have a personal valuation of the behavior. ${ }^{[34]}$ This requires patients to have autonomous, or internal motivation and a sense of competency, or confidence in the ability to change. Orem identified a "legitimate patient" as someone whose self-care agency, defined as a complex set of capabilities that enables individuals to perform self-care, is not adequate to meet their therapeutic self-care demand. ${ }^{[33]}$ According to Taylor and Renpenning, human actions result from motivations, intention that reflect personal values and desires, and when self-care is performed, it contributes to improved health and well-being. ${ }^{[35,36]}$ Collaboration in the plan of care extends beyond nursing to other members of the health care team. Patient involvement in decision-making may be beneficial as both congruence of the health care provider and patient perception is vital for successful goal setting as a product of achieving self-care. ${ }^{[37]}$ MI which is discussed in a later section is considered as a way of "being with" patients that can help them navigate change. ${ }^{[38]}$

\subsection{Self-efficacy}

Unlike Ryan et al.'s theory, Bandura's theory indicates that self-efficacy, or the belief in one's ability to change, is sufficient to implement change. ${ }^{[39]}$ This is conceptualized as both the confidence to abstain from a behavior and the ability to resist temptation to engage in the behavior. These levels of confidence and temptation fluctuate as patients experience physical and mental changes in their everyday lives. When faced with a new health condition, a patient must first acknowledge the seriousness of the problem. In order to move towards positive change, there needs to be personal affirmation of hope for success to exercise control over events. For example, to avoid relapse, a person with a substance abuse disorder must have a positive attitude towards avoiding peers who engage in similar activity. Assessment of the patients perceived ability to engage in meaningful or pleasurable, sober activities should be addressed before engaging in change strategies related to daily activities. Language regarding self-efficacy may include the following statements from the patient: (a) "I believe that I am confident that I can do that"; (b) "when I am with my family who drinks I cannot resist having one glass of wine"; (c) that is beyond my control"; or (d) "that will be easy for me."

\subsection{Triggers of change}

Motivation is a multidimensional concept encapsulated by 3 popular phases that a patient is able, willing, and ready to change. Ability, denotes the level of preparation and requires skills, resources and confidence or self-efficacy to carry out the new behavior. Willingness refers to the importance a person places on change or the level of desire to make the change. It is important to note that it is possible to feel willing yet unable to change. The combination of willingness and ability does not guarantee change. A patient can be willing and able to change, yet not ready to change. The ready component is the final stage in which the patient finally makes the decision to change a particular behavior. The plausible explanation for being willing and able but not ready is determined by the relative importance of the change compared with other priorities in the patient's life. For example, a mother with diabetes may not be able to exercise due to a hectic life style of caring for small children. Instilling motivation requires engaging in strong patient-centered relationships that allows the clinician to have therapeutic conversation to help the patient to become able, willing, and ready to take action. ${ }^{[16]}$

\subsection{The Transtheoretical Model of Change}

The Transtheoretical Model (TTM) of change by Prochaska and DiClemente has emerged over the past two decades as one of the most prolific models in the behavioral health field. ${ }^{[40]}$ The change process has been conceptualized as a sequence of stages through which patients progress as they consider, initiate, and maintain a new behavior. This body of work provides understanding of the major dimension of change, processes of change, decisional balance and selfefficacy. It offers important constructs to explain the process of intentional behavior change. In the TTM, the five stages of change are precontemplation, contemplation, preparation, action, and maintenance. Use of the processes of change has been widely tested in a range of behaviors such as exercise adherence, care planning, and safe sexual behavior. ${ }^{[41-43]}$ Clinicians can provide support to patients during any stage by using appropriate motivational strategies that are specific to the change stage that the client is in. The change process is cyclical, and many patients move back and forth between the stages at different rate. When a new problem is diagnosed it is not uncommon for the patient to linger in the early phases before plans are formalized to move into action. It is also important that as patients move into long term maintenance, there may be cycling through the different stages multiple times before adherence or stable change is achieved. The five stages are described in Figure 2. 

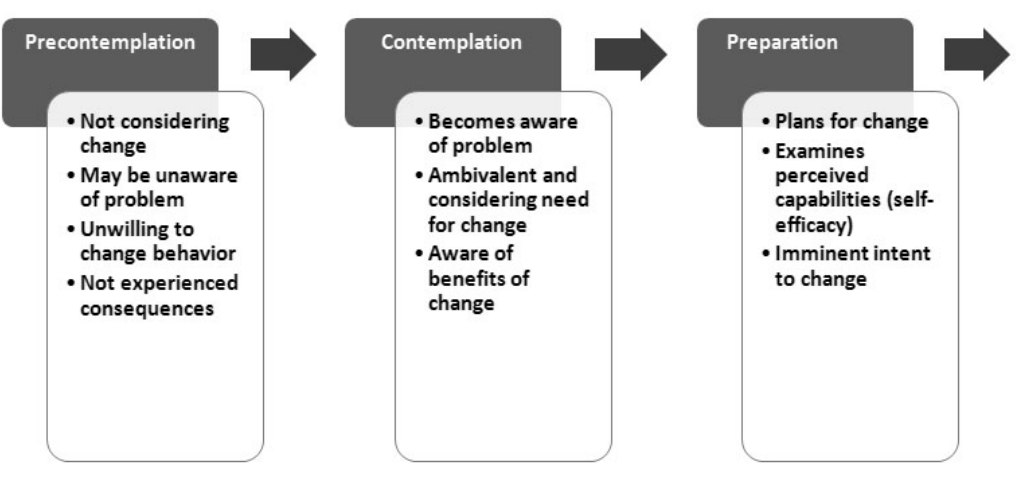

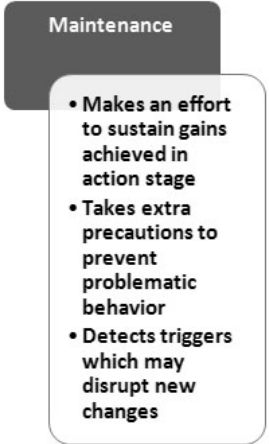

Figure 2. Stages of change

\subsection{Motivational Interviewing}

Motivational Interviewing (MI) is used to facilitate the change process and specific interventions are used at each stage of change to facilitate movement to the next stage. MI is a patient-centered approached to elicit intrinsic motivation for change by exploring and resolving ambivalence toward certain behaviors such as substance abuse and medication non-adherence. ${ }^{[38]}$ The process focuses on the patient's current concerns and is considered a way of being with the patient. The 3 concepts comprising the spirit of MI include collaboration, evocation, and autonomy. MI promotes a positive and supportive environment where the provider explores the patient's values rather than coerce or persuade. In MI, the provider should evoke concepts of change from the patient rather than acting as the expert or educator. The provider's role is to draw insight and motivation for change from the client. MI has four overarching principles: 1) express empathy, 2) develop discrepancy, 3) roll with resistance, and 4) support self-efficacy. In MI, the provider accepts the patient's ambivalence as a normal attitude. MI aims to develop discrepancy between current behaviors and goals/values. For example, if a patient is not adherent to medications but wants to reduce symptoms of congestive heart failure such as edema, the must identify this discrepancy in order to be motivated to adhere to medications. The patient rather than the provider should identify and present reasons for change (e.g., symptom reduction, staying outside of the hospital, improving quality of life, etc.). During an MI session, the patient may resist change. Instead of arguing for change, the provider should roll with the resistance. Advice or suggestions may be offered with permission from the patient, but not imposed on the patient. The last principle of MI, support self-efficacy, promotes the idea that the patient is responsible for the change and that the patient's belief of this contributes to the motivation to change.

The need for the unique and empathic communication style of MI in health care arises from the concept that a person's behavioral health impacts their physical health conditions and vice versa. ${ }^{[44]}$ A depressed patient with multiple medical comorbidities may have underlying psychosocial issues that affect the ability or motivation to adhere to medications or attend follow-up appointments. In a study of patients who experienced a cardiovascular event, depressed patients were more likely to not adhere to their medications or other medical instructions to prevent another acute coronary syndrome. ${ }^{[45]}$ To identify and address underlying issues affecting healthrelated behaviors, a communication style aimed at facilitating patient-driven discovery of underlying motivation to change health behaviors may be helpful. ${ }^{[4]}$ In MI, patients' beliefs about health behaviors including medications and other treatments are explored. Impact of MI on different outcomes such as readmission rates, biological markers (e.g., hemoglobin A1c, cholesterol levels), and medication adherence have been evaluated in the literature. In a study of 303 hospitalized adults comparing usual care (medication education) and MI, MI was a negative predictor of 30-day readmissions. ${ }^{[46]} \mathrm{A}$ systematic review of 37 studies utilizing MI techniques and spirit in the context of health behaviors identified studies with statistically significant evidence that MI positively influences health outcomes including physical activity, body weight, hemoglobin A1c, and cholesterol levels. ${ }^{[47]}$ A systematic review and meta-analysis of 48 randomized controlled trials examining MI in medical care settings found that MI had significant positive impact on blood pressure, cholesterol, HIV viral load, amount of alcohol consumption, tobacco abstinence, and marijuana use. ${ }^{[48]}$ Due to barriers including geographic, financial, and transportation, patients may not be able to frequently access in-person health care services. A systematic review of 9 studies using telephone-based MI for medication adherence in patients with various disease states including HIV, osteoporosis, mental illness, and diabetes found that 6 studies reported statistically significant differences between MI and control group for medication adherence changes. ${ }^{[49]}$ 


\section{MethodS}

\subsection{Pathway through the Behavioral Health Transition of Care Program}

The inclusion criteria for the program are 1) high risk for readmission (Discharge Decision Support System [D2S2] score of 3 or higher at admission) and 2) current or present mental health diagnosis, suicide attempt, or alcohol/drug abuse. Criterion number 2 is defined by the patient answering "yes" to the nurse-delivered question: Do you currently have or do you have a history of mental health diagnosis, suicide attempt, or alcohol or drug abuse? Patients who answer "no" to the above question are not asked the D2S2 questions unless there is a change in their psychiatric condition and/or new information about a mental health condition is obtained (e.g., a psychiatric consult entered for suicidal ideation). The D2S2 has been exclusively licensed to RightCare by the University of Pennsylvania. ${ }^{[50]}$ The tool is used by the inpatient unit, observation unit, and ED nurses and includes questions about symptoms of depression, cognitive status, comorbid conditions and caregiver availability. As the program progresses, social workers and even some physicians are beginning to engage in the D2S2 assessment process to ensure that patients are being risk stratified. The RightCare program calculates a score based on the answers to the tool's questions and other demographic data such as zip code. Patients scoring 3 or higher (range: 0-11) have a high risk for 30-day readmission or ED visit. Social workers interview, conduct assessments including the 8-item Morisky Medication Adherence Scale (MMAS-8) as well as provide disease self-management tools in patients that fit the program inclusion criteria. ${ }^{[51-53]}$ MMAS-8 is validated in elderly outpatients with hypertension and was found to have a sensitivity of $93 \%$, specificity of $53 \%$, and alpha reliability of $0.83 .{ }^{[51]}$ In the validation study, there was a significant relationship between blood pressure control and MMAS-8 score, thus making it a practical measure of adherence. Patients with MMAS- 8 score less than 6 have low adherence, 6 to less than 8 have medium adherence, and 8 have high adherence. If a patient scores low on the MMAS-8, the pharmacist is consulted to explore medication adherence issues with the patient. If the patient consents, the social worker enrolls the patients in the post-discharge services: 1 to 2 home visits with the nurse practitioner and home health aide within 30 days after discharge and/or automated telephone calls using Emmi Solutions Behavioral Health Questionnaire. ${ }^{[54]}$ Emmi Solutions' interactive web-based programs and call campaigns deliver actionable health information to patients via multiple modalities at key moments across the continuum of care. The program facilitated in development of the Behavioral Health questions for the Emmi Solutions calls. Within one to two days after discharge, the automated phone call asks the patient various questions related to psychiatric symptoms, medications, and follow-up appointments. The program receives a daily report of "Red Flags" revealed by the automated telephone calls where patients may report medication side effects and access issues, lack of follow up, and depressed mood. The program's nurse and psychologist, also known as educators, follow up on "Red Flag" patients. The home visits are conducted by the home health aide, and the nurse practitioner uses Face Time to communicate with the patient. See Figure 3 for the pathway through the program.

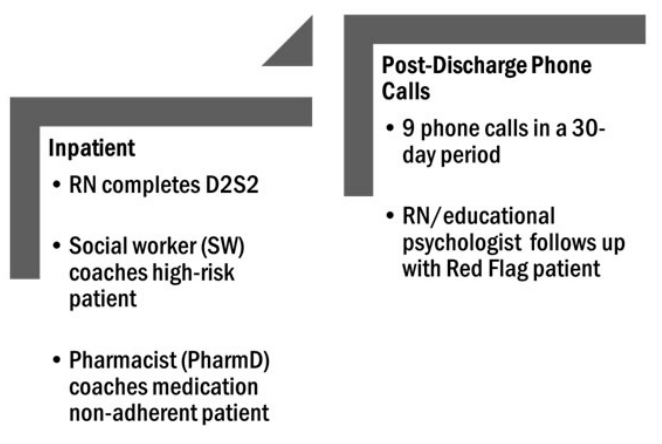

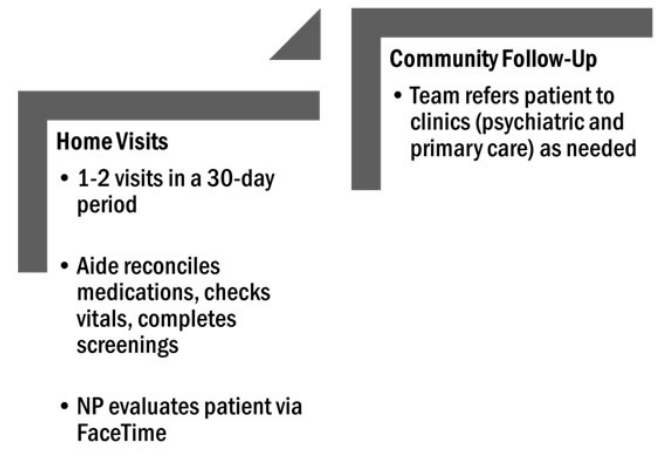

Figure 3. Behavioral health transition of care program pathway

\subsection{Coleman tools}

Coleman's four pillars are addressed by the program using the TBM. The social workers provide patients with a Per- sonal Health Record (PHR). Patients and their families are taught to be aware of medical and psychiatric symptoms, and know that if they experience new or more intense symptoms, 
they should call their physicians immediately. Team members frame this instruction as if patients are experts in their healthcare. High risk patients are seen by a social worker, whether they join the program or not, and receive a PHR. The PHR contains useful information on the patient's medical conditions and Red Flags and aims to empower the patient in managing their symptoms and to better engage with the rest of their health care team including physicians. Outpatient providers' contact information are listed along with their telephone numbers. Using these tools, the patient is empowered to conduct self-care.

\subsection{The Teach Back Method}

All program staff use TBM in some form to ensure patient understanding of health concepts such as disease selfmanagement, medications, and follow-up instructions. Social workers conduct the TBM after collaborating with patients on the PHR. The pharmacist conducts the TBM after reviewing medications or explaining complex medication instructions such as insulin doses or medication tapers. In the post-discharge phase, the TBM is used to reinforce teaching done in the inpatient phase. Staff also use TBM to ensure understanding of the date of doctor's appointments or dialysis.

\subsection{Motivational Interviewing}

Program staff including social workers, nurse practitioners, psychologist, pharmacist, nurse, and chemical dependency counselor attended a three-day MI training to formally learn how to engage patients, explore health-related concerns, and elicit behavior change. The need for this training stemmed from the complex psychosocial issues interfering with health behaviors such as medication adherence and timely follow-up with primary care physicians and psychiatrists. In practice, the program's staff reveal underlying maladaptive health behaviors including medication non-adherence, lack of followup, missed dialysis appointments, psychosocial issues and substance abuse that may contribute to emergency department visits and hospital readmissions. Through MI techniques, staff explore these issues with the patients and identify behavior change goals. The staff participating in the post-discharge phase follows up with the patient based on the stage of change.

\subsection{Program performance indicator calculation}

The reduction of the ED visit rate of patients with a behavioral health or substance abuse diagnosis is a program performance indicator. The one-year goal of the Behavioral Health Transition of Care Program is to reduce the ED visit rate of behavioral health patients by $5 \%$ from baseline (October 2013 through April 2014) to first year (April 2014 through March 2015). See Figure 4 for calculations.

$$
\begin{aligned}
& \frac{E D \text { visits for adults with behavioral health }(B H) \text { or substance abuse }(S A) \text { diagnosis }}{\text { Total number of } E D \text { visits for adults }} \times 100=B H / S A E D \text { visit rate } \\
& \frac{\text { Baseline } B H / S A E D \text { visit rate }- \text { First Year } B H / S A E D \text { visit rate }}{\text { Baseline BH/SA ED visit rate }} \times 100=B H / S A \text { ED visit rate reduction }
\end{aligned}
$$

Figure 4. Program performance indicator calculation

\section{Results}

Results are only reported for HMH and SJ. Since the inception of the project in June 2014, 2,330 encounters have been stratified as high risk for readmission or ED revisit using the D2S2. The average age of the high risk encounters is about 55 years old (range: $18-100$ years old) and about $5 \%$ of these encounters had Medicaid. The average D2S2 score is 4.4, or on the lower end of the high risk spectrum (3-11). The average MMAS- 8 score was 6.29 (medium adherence, range: 0-8). Approximately one-third of patients with a MMAS-8 score have low adherence. Social workers have provided patient-centered PHRs to about $74 \%$ of the high risk encounters and the nurse practitioners and home health aides

Published by Sciedu Press have seen about $13 \%$ of these high risk encounters in their home. Almost two-thirds of patients with a low MMAS-8 at $\mathrm{HMH}$ have been coached by the clinical pharmacist. $\mathrm{HMH}$ and SJ experienced a $4.6 \%$ reduction in the ED visit rate of behavioral health patients from April 2014 through March 2015. See Table 1 for a summary of the results. Due to data availability, there are differences in date ranges for the data items. A monthly performance trend of the behavioral health/substance abuse ED visit rate is in Figure 5. RightCare, the D2S2 vendor, reported data for the high risk patient populations at HMH and SJ from July 2014 through February 2015. From this patient population, $51.9 \%$ report depression and $43.1 \%$ report a loss of pleasure which RightCare links to lack of motivation. 
Table 1. Results for HMH and SJ (Variable Dates)

\begin{tabular}{|c|c|c|}
\hline Characteristic & $\mathbf{N}$ & $\%$ \\
\hline Total High Risk Encounters* & 2,330 & \\
\hline Female* & 1,371 & 58.84 \\
\hline Average Age (years)* & 55.8 years $(18-100)$ & \\
\hline Medicaid* & 109 & 4.68 \\
\hline Uninsured* & 223 & 9.57 \\
\hline Mean D2S2 Score (range: 0-11) (points)* & 4.4 & \\
\hline Mean MMAS-8 Score (range: 0-8) (points)\# & 6.29 & \\
\hline Encounters with Low MMAS-8 Score $(<6) \#, \dagger$ & 490 & 32.01 \\
\hline \multicolumn{3}{|l|}{ Performance Characteristic } \\
\hline Personal Health Records (PHR) Completed* & 1,715 & 73.61 \\
\hline Encounters with Home Visits Completed* & 314 & 13.48 \\
\hline Encounters Coached by Pharmacist\#, $\ddagger$ & 279 & 63.27 \\
\hline Encounters Enrolled in Emmi§ & 683 & 31.33 \\
\hline \% BH/SA ED visits baseline (10/1/13-3/31/14†† & & 31.50 \\
\hline \% BH/SA ED visits goal (4/1/14-3/31/15)†† & & 29.92 \\
\hline \% BH/SA ED visits actual (4/1/14-3/31/15)†† & & 30.04 \\
\hline $\mathrm{BH} / \mathrm{SA}$ ED visit rate reduction & & 4.63 \\
\hline
\end{tabular}

*Data from June 2014 through March 19, 2015; \#Data from June 2014 through March 31, 2015 (N = 2,575 high risk encounters at HMH and SJ); $\dagger$ Denominator is $\mathrm{N}=1,531$ (encounters with a MMAS-8 score at HMH and SJ); $¥$ Pharmacist coaches patients at HMH only, denominator is Encounters with Low MMAS-8 Score at HMH (N = 441); §Data from Right Care database, for patients admitted from June 2014 through March 31 , 2015 (denominator, $\mathrm{N}=2,180$ high risk patients); the number is smaller than the total number of high risk encounters from June 2014 through March 19 , 2015 because RightCare data excluded patients with an admit date earlier than June 16, 2014; ††This percentage reflects the total number of patients with a primary or secondary behavioral health and/or substance abuse diagnosis that visit the ED out of the total number of adult ED visits at HMH and SJ. This is not reflective of only the high risk encounters.

Use of the (C)MMAS is protected by US copyright laws. Permission for use is required. A license agreement is available from Donald E. Morisky, ScD, ScM, MSPH, Professor, Department of Community Health Sciences, UCLA School of Public Health, 650 Charles E Young Drive South, Los Angeles, CA $90095-1772$.

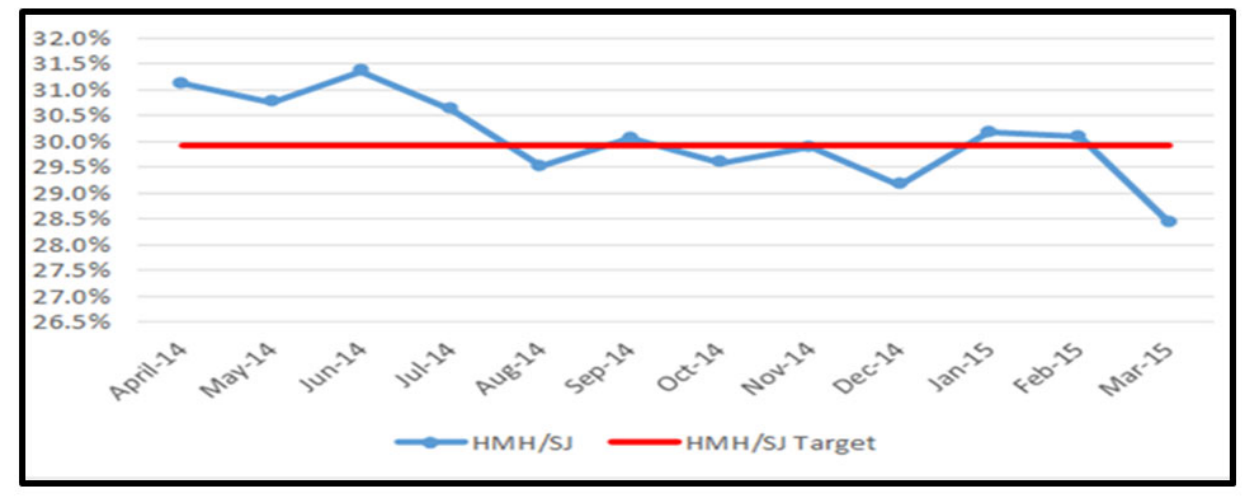

Figure 5. Monthly BH/SA ED visit rate

\section{Discussion}

The HMH Behavioral Health Transition of Care Program aims to reduce hospital readmissions and ED visits through empowering patients via various behavioral change and educational techniques. In an effort to curb unnecessary ED visits, the team targets patients with behavioral health and/or substance abuse disorders, since they are more likely to be heavy utilizers of the health care system. A major paradigm shift is required for the ED staff to address non-emergencies. The ED staff treat the chief complaint which may not necessarily prevent a patient from returning to the hospital in less than 30 days. In general, EDs are not designed to pro- 
vide talk therapy, health coaching or support poor social circumstances. This program is in the process of designing a paradigm shift in the ED and acute care units, in which staff take the time to use the D2S2 and collaborate with the Behavioral Health Transition of Care Program. For example, point of entry social workers stationed in the ED have been introduced to the program's tools. The program has identified multiple "super utilizers" to trend their utilization and conduct thorough chart reviews so that the team can help these patients manage their care outside the hospital. These cases require a coordinated effort among various health care providers including hospitalists, psychiatrists, and social workers.

Over 2,000 encounters were risk stratified over ten months and over $10 \%$ of these encounters have Medicaid or are uninsured. Patients who are uninsured and have mental health/substance abuse disorders may have socioeconomic limitations and difficulty navigating the health care system including obtaining services such as financial and social assistance from the city or county. These patients may benefit from additional guidance from the Behavioral Health Transition of Care Program including MI and practical guidance as well as repetitive TBM education on medications. Followup telephone calls may encourage this patient population to maintain self-care. Based on Right Care's data, many of these high risk patients are depressed or unmotivated, further supporting the programs' work to use MI to impact healthrelated behaviors. The programs' findings demonstrate that approximately one-third of the patients scored low on the MMAS-8 in the hospital, similar to the proportion (32.1\%) reported in a validity study of the MMAS- 8 in outpatients with hypertension. ${ }^{[51]}$ Barriers to medication adherence in the high risk encounters include intentional non-adherence (e.g., negative beliefs about medications), unintentional nonadherence (e.g., knowledge deficit, forgetfulness), substance abuse, financial barriers, logistical issues (e.g., no transportation to pharmacy), and no prescriber. The clinical pharmacist conducted face-to-face coaching for approximately two-thirds of the HMH patients with a low MMAS-8 score. MI techniques are used to engage patients and qualitative interviewing techniques are employed to identify barriers to medication non-adherence versus simply labeling the patient as "noncompliant". MI has been useful in this population to establish rapport with patients and elicit change talk. Future studies could investigate the impact of the clinical pharmacist on the MMAS-8 score after discharge.

More than $70 \%$ of the high risk encounters were provided PHRs to facilitate the self-care process at home. The Coleman Model provides a strategy for transitioning patients into a safer discharge. With this model in place, patients may feel

Published by Sciedu Press less likely that they have been "abandoned" by the inpatient team, or discharged too soon. One of the aims of MI and TBM is to increase patients' and caregivers' readiness to provide self-care. MI and TBM should be especially effective in respectively exploring health-related concerns and instructing patients and families about medications.

Of the high risk encounters, over 10\% had a home visit. Prior to initiation of this program, a baseline home visit rate was not established as this is a novel service provided by HMH in this population. Various contributing factors may impact the home visit rate including mistrust of strangers entering the home, patients' limited access to phones (e.g., disconnected phone lines), and a poor understanding of the home visit services (e.g., misconception that the program costs money). Some patients already have home health care, therefore they decline additional services. Logistical contributing factors include staff schedule limitations (i.e., social workers' ability to encounter each high risk encounter and offer them the program's services). Also, some patients leave against medical advice barring the social worker from introducing the program's services to these patients prior to discharge. Interdisciplinary group discussions are intermittently held to identify reasons for home visit refusals or no-shows. Social workers in the program use MI techniques to elicit patients to provide reasons to participate in this program, especially for those who declined the service in the past. Methods to inform patients on the home visit services include flyers and verbal descriptions. Roughly one-third of high risk patients admitted to HMH and SJ from June 2014 to March 2015 were enrolled in Emmi indicating that they are interested in receiving follow-up telephone calls from staff. Patients may feel more comfortable with a telephone call versus having a home visit.

CMS requires that the program report $\mathrm{BH} / \mathrm{SA}$ ED visit rate on all of the patients that enter the participating hospitals' EDs and not just the high risk encounters stratified by the program. The thought is that the more encounters the program stratifies as high risk and intervenes on, the lower the BH/SA ED visit rate would be. The 1-year percentage reduction goal for the ED visit rate is $5 \%$ and the 2-year goal is $10 \%$. For April 2014 through March 2015, the program achieved its goal $\mathrm{BH} / \mathrm{SA}$ ED visit rate reduction by $75 \%$. A variety of contributing factors may impact the $\mathrm{BH} / \mathrm{SA} \mathrm{ED}$ visit rate. The high demand and limited supply of PCPs may lead to ED utilization for non-urgent issues like prescription refills. ${ }^{[9]}$ Furthermore, expansions of insurance coverage through the Affordable Care Act may contribute to high levels of ED usage as well. ${ }^{[55,56]}$

The Behavioral Health Transition of Care Program has var- 
ious strengths and challenges. The program consists of a multidisciplinary team that is trained to address the needs of this high risk population. The work done by the team aims to care for an underserved population that lacks holistic or coordinated discharge planning. The novel use of assessment tools and technologies (e.g., Emmi automated telephone services, risk stratification tool, MMAS-8, and iPads). Furthermore, the program sheds light on the behavioral health population's socioeconomic issues that impact their ability to care for themselves after discharge. Through MI and TBM, the team identifies patients' barriers to medication adherence and disease management. They communicate these barriers to self-care to the patients' primary team. The program at $\mathrm{HMH}$ has begun to change the culture at $\mathrm{HMH}$ and has collaborated with ED staff to form an ad hoc team to address "super utilizers". Challenges of the program include increasing high risk stratification by the unit nurses and post-discharge services enrollment. Nurses trained on the risk stratification tool may not recall how to access the tool in the electronic medical record or are busy addressing urgent issues. Through several rounds of education on the purpose of the tool, the team has aimed to communicate to the nurses that the goal of the risk stratification tool is to identify patients who may benefit from additional help from the transition of care team. Patients, occasionally confused by the hospitalization and discharge processes, may agree to enrolling in the post-discharge services without fully realizing that the transition of care program centers on telephone calls and a home visit rather than traditional home health care. Focus groups with patients and discussions among the interdisciplinary program team are conducted to determine how to increase enrollment in the post-discharges services offered by the program. The educators and nurse practitioners who commonly interact with patients after discharge emphasize the program's benefits. A number of patients expressed reluctance in having the aides come into their homes, nervous about the experience and fearful of having their residences judged. The team assures them the visit will be as non-intrusive as possible, and is not a social call. Another limitation in regards to data collection is difficulty in teasing out data for individual hospitals. This limitation is being addressed through collaboration with RightCare, Emmi and the hospital's electronic medical record support. Regardless, this preliminary data gives insight into the program's productivity and patient characteristics.

\section{Conclusions}

The Behavioral Health Transition of Care Program, a DSRIP 1115 Waiver program, is currently active at three HMH system hospitals. This paper reports two of the three hospitals' data with a favorable outcome over a ten month time period. Thus far, the high risk patients stratified by the D2S2 generally lack motivation, report depression, and have moderate medication adherence scores deeming this population to be challenging as they require focused efforts to address needs. The program uses MI, TBM and the Coleman Model as behavioral change and educational techniques to impact self-care in the behavioral health population. A majority of the high risk encounters have been coached or counseled by the program's staff employing a combination of these techniques in efforts to educate and subsequently lower the BH/SA ED visit rate. The program's performance indicator, $\mathrm{BH} / \mathrm{SA}$ ED visit rate reduction, indicates there has been a $4.63 \%$ reduction in $\mathrm{BH} / \mathrm{SA} \mathrm{ED}$ visit rates. By instituting a transitional care program, patients who score at high risk of returning to the hospital can be offered the chance to have follow-up telephone calls and home visits post-discharge as the program strives to keep targeted patients out of the hospital for 30 days. Overall, this innovative program provides novel transition of care services to a population who was traditionally overlooked due to preconceived notions regarding noncompliance and limited knowledge of the care team regarding community resources to support transitions in care.

\section{CONFlicts OF INTEREST Disclosure}

The authors declare that there is no conflict of interest.

\section{REFERENCES}

[1] Rau J. Medicare fines 2,610 hospitals in third round of readmission penalties. Kaiser Health News. 2014 October. Available from: http://kaiserhealthnews.org/news/medicare-rea dmissions-penalties-2015

[2] Coleman EA, Boult CE. Improving the quality of transitional care for persons with complex care needs. J Am Geriatr Soc. 2003; 51: 5567. PMid:12657079 http://dx.doi.org/10.1046/j.1532-541 $5.2003 .51186 . x$

[3] De Hert M, Schreurs V, Vancampfort D, et al. Metabolic syndrome in people with schizophrenia: a review. World Psychiatry. 2009; 8: 1522. http://dx.doi.org/10.1002/j.2051-5545.2009.tb001 99. $\mathrm{x}$

[4] Luhrmann TM. Of two minds: the growing disorder in American psychiatry. 1st ed. New York: Knopf; 2000. 337 p.

[5] Kronish IM, Rieckmann N, Halm EA, et al. Persistent depression affects adherence to secondary prevention behaviors after acute coronary syndromes. J Gen Intern Med. 2006; 21: 117883. PMid:16899061 http://dx.doi.org/10.1111/j.1525-1 $497.2006 .00586 . x$ 
[6] Texas Medicaid \& Healthcare Partnership. Potentially preventable readmissions in the Texas Medicaid population. Austin (TX): Health and Human Services Commission; 2012 Nov. 58 p.

[7] Burton R. Health policy brief: care transitions. Health Aff. 2012 Sep. Available from: http://www.healthaffairs .org/health policybriefs/brief .php?brief_id=76

[8] Gindi RM, Cohen RA, Kirzinger WK. Emergency room use among adults aged 18-64: Early release of estimates from the National Health Interview Survey, January-June 2011. National Center for Health Statistics (US). 2012 May. Available from: http://www.cd c.gov/nchs/nhis/releases.htm

[9] Usher-Pines L, Pines J, Kellerman A, et al. Deciding to visit the emergency department for non-urgent conditions: a systematic review of the literature. Am J Manag Care. 2013; 19(1): 47-59.

[10] O'Shea JS. The crisis in America's emergency rooms and what can be done. The Heritage Foundation. 2007 December. Available from: http://s3. amazonaws. com/thf_media/2007/pdf/ bg2092.pdf

[11] Simoneau H, Bergeron J. Factors affecting motivation during the first six weeks of treatment. Addict Behav. 2003; 28: 1219-41. http://dx.doi .org/10.1016/S0306-4603(02) 00257-5

[12] Hyde YM, Kautz DD. Enhancing health promotion during rehabilitation through information-giving, partnership-building, and teachback. Rehabil Nurs. 2014; 39: 178-82. PMid:24037997 http: //dx.doi.org/10.1002/rnj.124

[13] Wasson J, Coleman EA. Health confidence: A simple, essential measure for patient engagement and better practice. Fam Pract Manag. 2014; 21: 8-12. PMid:25251348

[14] Coleman EA. The Care Transitions Program $®$ Structure [Internet]. Denver (CO): University of Colorado, School of Medicine, Division of Health Care Policy and Research; 2007. Available from: http://www.caretransitions.org/structure.asp

[15] Ali S, Getz MJ, Chung H. Bridging the gap for patients with mental illness. Ment Health Clin. 2015; 5(1): 40-5. http://dx. doi .org $/ 10.9740 / \mathrm{mhc} .2015 .01 .040$

[16] Center for Substance Abuse Treatment. Enhancing Motivation for Change in Substance Abuse Treatment. Treatment Improvement Protocol (TIP) Series, No. 35. HHS Publication No. (SMA) 13-4212. Rockville, MD: Substance Abuse and Mental Health Services Administration. 1999.

[17] American Psychiatric Association (APA). Diagnostic and Statistical Manual of Mental Disorders, 4th Edition, Text Revision. Washington, DC: APA. 2000.

[18] Berwick D. What 'patient-centered' should mean: confessions of an extremist. Health Aff. 2009; 28(4): w555-w565. PMid:19454528 http://dx.doi.org/10.1377/hlthaff.28.4.w555

[19] Velasquez M, Sternberg KV, Dodrill CL, et al. The transtheoretical model as a framework for developing substance abuse interventions. J Addict Nurs. 2005; 16: 31-40. http://dx. doi .org/10.1080/1 0884600590917174

[20] Sax G. Principles of educational and psychological measurement and evaluation. 4th ed. Belmont, CA: Wadsworth, 2010. 688 p.

[21] Prochaska JO, Norcross JC. Systems of psychotherapy: A transtheoretical analysis. 7th ed. Boston, MA: Brooks-Cole; 2009. 624 p.

[22] Cohen MZ, Jenkins D, Holston EC, et al. Understanding health literacy in patients receiving hematopoietic stem cell transplantation. Oncol Nurs Forum. 2013; 40: 508-15. PMid:23989024 http://dx.doi.org/10.1188/13.0NF.508-515

[23] Maykovich MK. Medical sociology. Sherman Oaks, CA: Alfred; $1980.554 \mathrm{p}$.

Published by Sciedu Press
[24] Warner R. Morality in medicine: An introduction to medical ethics Sherman Oaks, CA: Alfred. 1980. 142 p.

[25] Coleman EA, Parry C, Chalmers S, et al. The care transitions intervention: results of a randomized controlled trial. Arch Intern Med 2006; 166: 1822-8. PMid:17000937 http://dx.doi.org/10.10 01/archinte.166.17.1822

[26] Coleman EA, Chugh A, Williams MV, et al. Understanding and execution of discharge instructions. Am J Med Qual. 2013; 28: 383 91. PMid:23354870 http://dx.doi.org/10.1177/106286061 2472931

[27] White M, Garbez R, Carroll M, et al. Is "teach-back" associated with knowledge retention and hospital readmission in hospitalized heart failure patients? J Cardiovasc Nurs. 2013; 28: 13746. PMid:22580624 http://dx.doi.org/10.1097/JCN.0b013 e31824987bd

[28] Kripalani S, Bengtzen R, Henderson LE, et al. Clinical research in low-literacy populations: Using teach-back to assess comprehension of informed consent and privacy information. IRB: Ethics and Human Research. 2008; 30: 13-19. PMid:18512655

[29] Tamura-Lis W. Teach-back for quality education and patient safety. Urol Nurs. 2013; 33: 267-71, 298. PMid:24592519

[30] Tamariz L, Palacio A, Robert M, et al. Improving the informed consent process for research subjects with low literacy: A systematic review. J Gen Int Med. 2012; 28: 121-6. PMid:22782275 http://dx.doi.org/10.1007/s11606-012-2133-2

[31] Xu P. Using teach-back for patient education and self-management. American Nurse Today. 2012 March. Available from: http://www. americannursetoday.com/using-teach-back

[32] Black JT, Romano PS, Banafsheh S, et al. A remote monitoring and telephone nurse coaching intervention to reduce readmissions among patients with heart failure: study protocol for the Better Effectiveness after Transition - Heart Failure (BEAT-HF) randomized controlled trial. Trials. 2014; 15: 124. PMid:24725308 http://dx.doi.org/10.1186/1745-6215-15-124

[33] Orem D. Nursing: concepts of practice. 4th ed. St. Louis: Mosby; 1990. 385 p. PMid:2198591

[34] Ryan R, Patrick H, Deci D, et al. Facilitating health behavior change and its maintenance: interventions based on self-determination theory. The European Psychologist. 2008; 10: 2-5.

[35] Lee M. Power, self-care and health in women living in urban squatter settlements in Karachi, Pakistan: a test of Orem's theory. J Adv Nurs. 1999; 30(1): 248-59. http://dx.doi.org/10.1046/j.1365-2 $648.1999 .01070 . \mathrm{x}$

[36] Taylor S, Renpenning K. Self-care science, nursing theory, and evidence-based practice. 1st ed. New York: Spring Publishing Company; 2011. $256 \mathrm{p}$.

[37] Ward-Griffin C, Bramwell L. The congruence of elderly client and nurse perception of the client's self-care agency. J Adv Nurs. 1990; 15(9): 1070-7. PMid:2229706 http://dx.doi.org/10.1111/j .1365-2648.1990.tb01988.x

[38] Miller WR, Rollnick S. Motivational interviewing: preparing people for change. 2nd ed. New York: The Guilford Press; 2002. 390 p.

[39] Bandura A. The anatomy of stages of change. Am J Health Promot. 1997; 12(1): 8-10. PMid:10170438 http://dx.doi.org/10.42 78/0890-1171-12.1.8

[40] Prochaska JO, Diclemente CC. The transtheoretical approach: crossing traditional boundaries of therapy. Homewood, Illinois: Dow Jones-Irwin; 1994. 204 p.

[41] Huseb AM, Dyrstad SM, Sreide JA, et al. Predicting exercise adherence in cancer patients and survivors: a systematic review and meta-analysis of motivational and behavior factors. J Clin Nurs. 2013; 22(1-2): 4-21. 
[42] Fried TR, Redding CA, Robbins ML, et al. Stages of change for the component behaviors of advance care planning. J Am Geriatr Soc. 2010; 58(12): 2329-36. PMid:21143441 http://dx.doi.org/10. $1111 / j .1532-5415.2010 .03184 . x$

[43] Redding CA, Rossi JS, Rossi SR, et al. Health behavior models. The International Electronic Journal of Health Education. 2000; 3(Special Issue): 180-93.

[44] Sciacca K. A language for integrated care. Behav Healthc. 2012 September. PMid:23330305

[45] Kronish IM, Rieckmann N, Halm EA, et al. Persistent depression affects adherence to secondary prevention behaviors after acute coronary syndromes. J Gen Intern Med. 2006; 21(11): 1178 83. PMid:16899061 http://dx.doi.org/10.1111/j.1525-1 497.2006.00586.x

[46] Hyrkas K, Wiggins M. A comparison of usual care, a patientcentred education intervention and motivational interviewing to improve medication adherence and readmissions of adults in an acute-care setting. J Nurs Manag. 2014; 22: 350-61. PMid:24612391 http://dx.doi.org/10.1111/jonm.12221

[47] Copeland L, McNamara R, Kelson M, et al. Mechanisms of change within motivational interviewing in relation to health behaviors outcomes: a systematic review. Patient Educ Couns. 2015; 98: 40111. PMid:25535015 http://dx.doi.org/10.1016/j.pec. 201 4.11 .022

[48] Lundahl B, Moleni T, Burke BL, et al. MI in medical care settings: a systematic review and meta-analysis of randomized controlled trials. Patient Educ Couns. 2013; 93: 157-68. PMid:24001658 http://dx.doi.org/10.1016/j.pec.2013.07.012
[49] Teeter BS, Kavookjian J. Telephone-based motivational interviewing for medication adherence: a systematic review. Transl Behav Med. 2014; 4(4): 372-81. PMid:25584086 http://dx.doi .org/10 . 10 07/s13142-014-0270-3

[50] Right Care. Pennsylvania: RightCare Solutions, Inc.; 2015. Available from: http: //www.rightcaresolutions.com

[51] Morisky DE, Ang A, Krousel-Wood M, et al. Predictive validity of a medication adherence measure for hypertension control. J Clin Hypertens. 2008; 10: 348-54. http://dx.doi.org/10.1111/j $.1751-7176.2008 .07572 . \mathrm{x}$

[52] Krousel-Wood MA, Islam T, Webber LS, et al. New medication adherence scale versus pharmacy fill rates in seniors with hypertension. Am J Manag Care. 2009; 15(1): 59-66. PMid:19146365

[53] Morisky DE, DiMatteo MR. Improving the measurement of selfreported medication nonadherence: final response. J Clin Epidemio. 2011; 64: 258-63. http://dx.doi.org/10.1016/j.jclinepi. 2010.02 .023

[54] Emmi Solutions White Papers. Emmi Solutions; [cited 2015 May 22]. Available from: http://emmisolutions.mwnewsroom.com /White-Papers

[55] Hosseinichimeh N, Weinberg S. The effect of the Massachusetts healthcare reform on emergency department use. World Med Health Policy. 2014; 6(1): 9-21. http://dx.doi.org/10.1002/wmh3. 77

[56] Pitts S, Carrier E, Rich E, et al. Where Americans get acute care: increasingly, it's not at their doctor's office. Health Aff. 2010; 29(9): 1620-9. PMid:20820017 http://dx.doi.org/10.1377/hltha ff. 2009.1026 